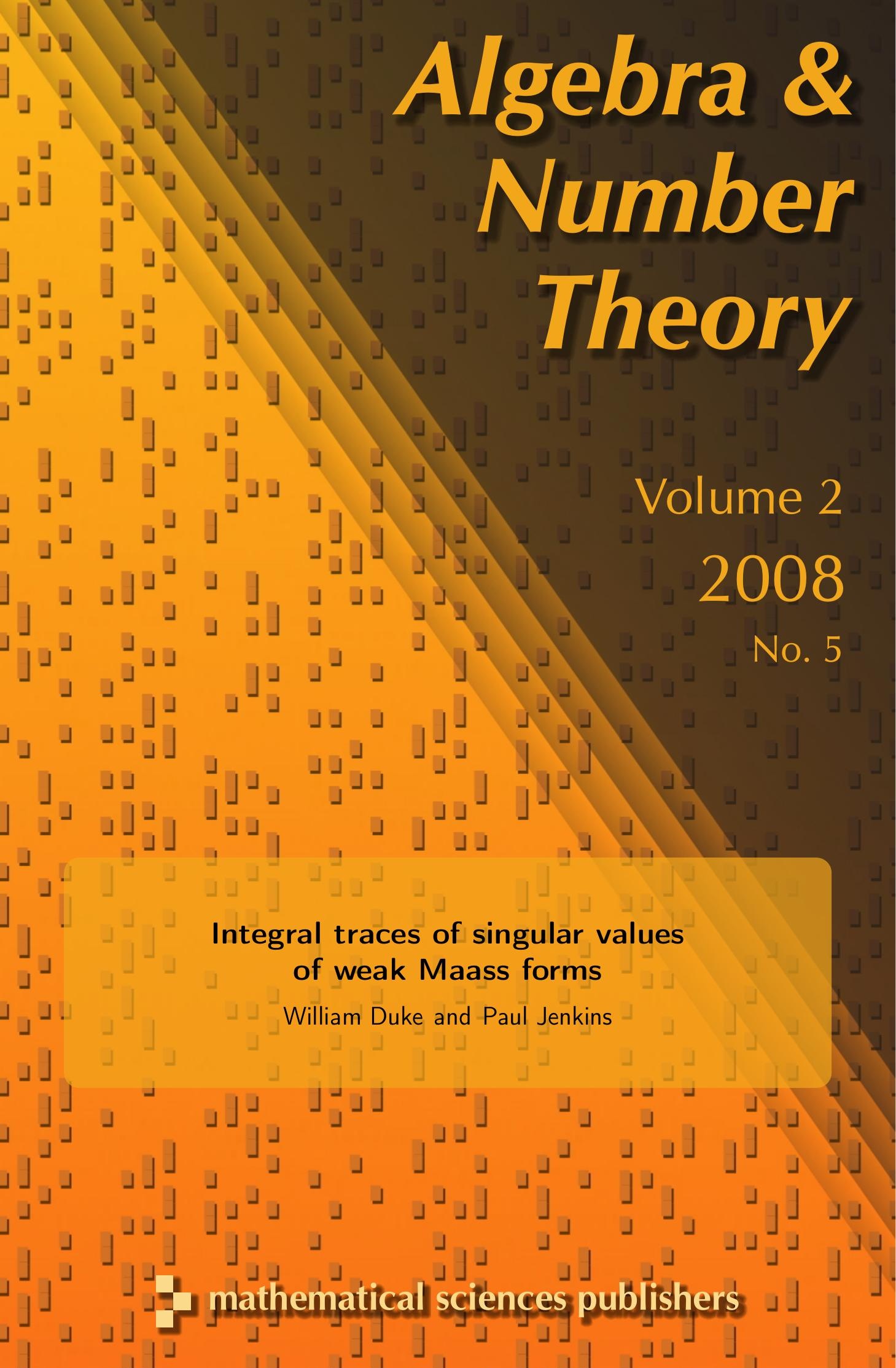




\title{
Integral traces of singular values of weak Maass forms
}

\author{
William Duke and Paul Jenkins
}

\begin{abstract}
We define traces associated to a weakly holomorphic modular form $f$ of arbitrary negative even integral weight and show that these traces appear as coefficients of certain weakly holomorphic forms of half-integral weight. If the coefficients of $f$ are integral, then these traces are integral as well. We obtain a negative weight analogue of the classical Shintani lift and give an application to a generalization of the Shimura lift.
\end{abstract}

\section{Introduction}

Recently there has been a resurgence of interest in the classical theory of singular moduli, these being the values of the modular $j$-function at quadratic irrationalities. This resurgence is due largely to the influential papers of Borcherds [1995; 1998] and Zagier [2002]. The present paper arose from a suggestion, made at the end of Zagier's paper, to extend some of the results given there on traces of singular moduli to higher weights. One such generalization has been given recently by Bringmann and Ono [2007], who provide an identity for the traces associated to certain Maass forms in terms of the Fourier coefficients of half-integral weight Poincaré series. However, it does not seem to be known when these traces are integral or even rational. Here we will identify the traces associated to a weakly holomorphic form $f$ of negative-integral weight with the coefficients of certain weakly holomorphic forms of half-integral weight and show that these coefficients are integral when the coefficients of $f$ are integral. We will use this identification to obtain a negative weight analogue of the classical Shintani lift. We also give an application to Borcherds's generalization of the Shimura lift to weakly holomorphic modular forms.

MSC2000: primary 11F30; secondary 11F37.

Keywords: weak Maass forms, weakly holomorphic modular forms, traces of singular moduli. Duke is supported by NSF grant DMS-0355564, and Jenkins is supported by NSF grant DMS0603271. 
Recall that a weakly holomorphic modular form of weight $k$, where $k \in 2 \mathbb{Z}$, is a holomorphic function $f$ on the upper half-plane $\mathcal{H}$ that satisfies

$$
\left(\left.f\right|_{k} \gamma\right)(\tau)=(c \tau+d)^{-k} f\left(\frac{a \tau+b}{c \tau+d}\right)=f(\tau) \quad \text { for all } \gamma=\left(\begin{array}{ll}
a & b \\
c & d
\end{array}\right) \in \Gamma=\operatorname{PSL}(2, \mathbb{Z})
$$

and that has a $q$-expansion $f(\tau)=\sum_{n} a(n) q^{n}$ with $a(n)=0$ for all but finitely many $n<0$; here, as usual, $q=e(\tau)=e^{2 \pi i \tau}$. Let $M_{k}^{!}$denote the vector space of all weakly holomorphic modular forms of weight $k$. Similarly, for $k=s+1 / 2$ with $s \in$ $\mathbb{Z}$, let $M_{k}^{!}$denote the space of holomorphic functions on $\mathcal{H}$ that transform like $\theta^{2 k}$ under $\Gamma_{0}(4)$, have at most poles in the cusps, and have a $q$-expansion supported on integers $n$ with $(-1)^{s} n \equiv 0,1(\bmod 4)$. Here, as usual, $\theta(\tau)=\sum_{n \in \mathbb{Z}} q^{n^{2}}$. For any $k$, let $M_{k} \subset M_{k}^{!}$denote the subspace of holomorphic forms and $S_{k} \subset M_{k}$ the subspace of cusp forms.

In this paper $d$ is always an integer with $d \equiv 0,1(\bmod 4)$, and $D$ is always a fundamental discriminant (possibly 1 ). Suppose $d D<0$ and $F$ is a $\Gamma$-invariant function on $\mathcal{H}$. Define the twisted trace

$$
\operatorname{Tr}_{d, D}(F)=\sum_{Q} w_{Q}^{-1} \chi(Q) F\left(\tau_{Q}\right)
$$

where the sum is over a complete set of $\Gamma$-inequivalent positive definite integral quadratic forms $Q(x, y)=a x^{2}+b x y+c y^{2}$ with discriminant $d D=b^{2}-4 a c$, and

$$
\tau_{Q}=\frac{-b+\sqrt{d D}}{2 a} \in \mathcal{H}
$$

is the associated CM point. Here $w_{Q}$ is equal to 1 unless $Q \sim a\left(x^{2}+y^{2}\right)$ or $Q \sim a\left(x^{2}+x y+y^{2}\right)$, in which case $w_{Q}$ is equal to 2 or 3 , respectively. Also

$$
\chi(Q)=\chi(a, b, c)= \begin{cases}\chi_{D}(r) & \text { if }(a, b, c, D)=1 \text { and } \\ & Q \text { represents } r, \text { where }(r, D)=1 ; \\ 0 & \text { if }(a, b, c, D)>1,\end{cases}
$$

where $\chi_{D}$ is the Kronecker symbol. It is known that $\chi$ is well defined on classes, that $\chi$ restricts to a real character (a genus character) on the group of primitive classes, and that all such characters arise this way.

For the usual $j$-function $j=E_{4}^{3} / \Delta \in M_{0}^{!}$with Fourier expansion

$$
j(\tau)=q^{-1}+744+196884 q+21493760 q^{2}+\cdots,
$$

it is classical that the value $j\left(\tau_{Q}\right)$ is an algebraic integer in an abelian extension of $\mathbb{Q}(\sqrt{d D})$. Let $j_{1}=j-744$. Zagier [2002] showed that for a fundamental 
discriminant $D \neq 1$ we have

$$
\begin{gathered}
q^{-|D|}+\sum_{d>0} d^{-1 / 2} \operatorname{Tr}_{d, D}\left(j_{1}\right) q^{|d|} \in M_{1 / 2}^{!} \quad \text { if } D<0, \\
q^{-|D|}-D^{-1 / 2} \sum_{d<0} \operatorname{Tr}_{d, D}\left(j_{1}\right) q^{|d|} \in M_{3 / 2}^{!} \quad \text { if } D>0,
\end{gathered}
$$

and that both forms have integral Fourier coefficients. For instance, when $D=-3$ and $D=5$ we have the two weakly holomorphic forms

$$
\begin{array}{r}
q^{-3}-248 q+26752 q^{4}-85995 q^{5}+\cdots \in M_{1 / 2}^{!} \text {and } \\
q^{-5}+85995 q^{3}-565760 q^{4}+52756480 q^{7}+\cdots \in M_{3 / 2}^{!},
\end{array}
$$

and $\operatorname{Tr}_{5,-3}\left(j_{1}\right)=\operatorname{Tr}_{-3,5}\left(j_{1}\right)=j\left(\frac{1+\sqrt{-15}}{2}\right)-j\left(\frac{1+\sqrt{-15}}{4}\right)=-85995 \sqrt{5}$.

In this paper we will give such a result when $j_{1}$ is replaced by a function $f$ of negative weight. To state it, first define the Maass raising operator $\partial_{k}$ in $\tau=x+i y$ :

$$
\partial_{k}=\mathcal{D}-\frac{k}{4 \pi y}, \quad \text { where } \mathcal{D}=\frac{1}{2 \pi i} \frac{d}{d \tau}=q \frac{d}{d q} .
$$

Now $\partial_{k}\left(\left.f\right|_{k} \gamma\right)=\left.\left(\partial_{k} f\right)\right|_{k+2} \gamma$ for any $\gamma \in \operatorname{PSL}(2, \mathbb{R})$. Thus, if $f \in M_{2-2 s}^{!}$for $s \in \mathbb{Z}^{+}$, the function $\partial^{s-1} f$ is $\Gamma$-invariant, where

$$
\partial^{s-1} \equiv(-1)^{s-1} \partial_{-2} \circ \partial_{-4} \circ \cdots \circ \partial_{4-2 s} \circ \partial_{2-2 s} .
$$

After Maass we know that $\partial^{s-1} f$ is an eigenfunction of the Laplacian

$$
\Delta=-y^{-2}\left(\frac{\partial^{2}}{\partial x^{2}}+\frac{\partial^{2}}{\partial y^{2}}\right)
$$

with eigenvalue $s(1-s)$, so $\partial^{s-1} f$ is a weak Maass form (see for example [Bruinier et al. 2008, page 162] for a precise definition). Using a method that readily generalizes, Zagier [2002] showed in special cases that $\partial^{s-1} f$ is a rational function of $j$ and $h=E_{2}^{*} E_{4} E_{6} / \Delta$, where

$$
E_{2}^{*}(\tau)=1-24 \sum_{n \geq 1} \sigma(n) q^{n}-\frac{3}{\pi y}
$$

is the nonholomorphic weight 2 Eisenstein series and $\sigma(n)=\sum_{m \mid n} m$. For a CM point like $\tau_{Q}$ given in (1), it was shown by Ramanujan [1914, Equation (23), page 33 ] that $h\left(\tau_{Q}\right)$ is algebraic. More precisely, $h\left(\tau_{Q}\right) \in \mathbb{Q}\left(j\left(\tau_{Q}\right)\right)$; see [Masser 1975, Theorem A1, page 114]. Using this, we can deduce the remarkable fact that for any $f \in M_{2-2 s}^{!}$with $s \geq 1$ and with rational Fourier coefficients, the "singular" value of the weak Maass form $\partial^{s-1} f\left(\tau_{Q}\right)$ is algebraic. We are thus motivated to study $\operatorname{Tr}_{d, D}\left(\partial^{s-1} f\right)$ for such $f$. 
For $D$ a fundamental discriminant, let $\hat{s}=s$ if $(-1)^{s} D>0$ and $\hat{s}=1-s$ otherwise. It is also convenient to set

$$
\operatorname{Tr}_{d, D}^{*}(f)=(-1)^{\lfloor(\hat{s}-1) / 2\rfloor}|d|^{-\hat{s} / 2}|D|^{(\hat{s}-1) / 2} \operatorname{Tr}_{d, D}\left(\partial^{s-1} f\right) .
$$

Suppose $f \in M_{2-2 s}^{!}$for $s \geq 2$ has Fourier coefficients $a(n)$. For $D$ fundamental, define the $D$-th Zagier lift of $f$ to be

$$
\begin{aligned}
\mathfrak{Z}_{D} f(\tau)=\sum_{m>0} a(-m) m^{s-\hat{s}} \sum_{n \mid m} \chi_{D}(n) n^{\hat{s}-1} q^{-m^{2}|D| / n^{2}} & \\
& +\frac{1}{2} L\left(1-s, \chi_{D}\right) a(0)+\sum_{d: d D<0} \operatorname{Tr}_{d, D}^{*}(f) q^{|d|} .
\end{aligned}
$$

The linear map $f \mapsto \mathfrak{Z}_{D}(f)$ is a negative weight analogue of the Shintani lift on integral weight cusp forms. This follows from our main result, whose proof will be completed in Section 5 .

Theorem 1. Suppose $f \in M_{2-2 s}^{!}$for an integer $s \geq 2$. If $D$ is a fundamental discriminant with $(-1)^{s} D>0$, we have that $\mathfrak{Z}_{D} f \in M_{3 / 2-s}^{!}$, while if $(-1)^{s} D<0$, then $\mathfrak{Z}_{D} f \in M_{s+1 / 2}^{!}$. If $f$ has integral Fourier coefficients, then so does $\mathfrak{Z}_{D} f$.

Here we will not treat the case $s=1$, which requires special considerations and which can be dealt with by the methods of [Zagier 2002]. Furthermore, when $s=2,3,4,5,7$, Theorem 1 can also be deduced from results of [Zagier 2002]. The first new example occurs when $s=6$ and $D=1$, where we have the pair

$$
\begin{aligned}
f(\tau) & =E_{14}(\tau) / \Delta(\tau)^{2}=q^{-2}+24 q^{-1}-196560-47709536 q+\cdots \in M_{-10}^{!}, \\
\mathfrak{Z}_{1} f(\tau) & =q^{-4}+56 q^{-1}+390+15360 q^{3}+42264 q^{4}+615240 q^{7}+\cdots \in M_{-9 / 2}^{!} .
\end{aligned}
$$

Here $-\frac{1}{2} \zeta(-5) \cdot 196560=390$ and the first few values of $\operatorname{Tr}_{d, 1}^{*}(f)$ are

$$
3^{-4} \partial^{5} f\left(\frac{1+\sqrt{-3}}{2}\right)=15360, \quad 2^{-7} \partial^{5} f(i)=42264, \quad 7^{-3} \partial^{5} f\left(\frac{1+\sqrt{-7}}{2}\right)=615240 .
$$

Similarly, when $D=-3$ we have

$$
\mathfrak{Z}_{-3} f(\tau)=2^{11} q^{-12}-8 q^{-3}-15360 q-53319598080 q^{4}+\cdots \in M_{13 / 2}^{!} .
$$

The main new difficulty in proving Theorem 1 comes from the existence of cusp forms in $M_{2 s}^{!}$. The method of Poincaré series adapts nicely to handle it. A key dividend of the method is the last statement of Theorem 1, showing that the integrality of coefficients is preserved under the lift.

Remarks. First, it follows from Theorem 1 that if $(-1)^{s} D>0$, then the image $\mathfrak{Z}_{D}(f) \in M_{3 / 2-s}^{!}$is determined by its principal part and hence by the principal part of $f$. Furthermore, $a(0)$ is divisible by the denominator of each of the $L$-values $\frac{1}{2} L\left(1-s, \chi_{D}\right)$, provided that the Fourier coefficients of $f$ are integral. Using wellknown properties of the generalized Bernoulli numbers, one can reproduce the 
divisibility properties that follow from work of Siegel [1969, pages 254-256]. On the other hand, if $(-1)^{s} D<0$ then $\frac{1}{2} L\left(1-s, \chi_{D}\right)=0$.

Next, it can be shown that the Zagier lift is compatible with the Hecke operators. For details, see the end of Section 5.

Finally, using a theta lift, Bruinier and Funke [2006] have generalized Zagier's result in various other ways, for instance to higher levels, where the existence of cusp forms in the dual weight is also a complication; see also [Funke 2007].

As another application of these methods, we will give a simple proof of a basic property of the Shimura lift for weakly holomorphic modular forms. For

$$
g(\tau)=\sum_{n} b(n) q^{n} \in M_{s+1 / 2}^{!}
$$

with $s \in \mathbb{Z}^{+}$and $D$ fundamental with $(-1)^{s} D>0$, define the $D$-th Shimura lift of $g$ by

$$
\mathscr{Y}_{D} g(\tau)=\frac{1}{2} L\left(1-s, \chi_{D}\right) b(0)+\sum_{m>0}\left(\sum_{n \mid m} \chi_{D}(n) n^{s-1} b\left(m^{2}|D| / n^{2}\right)\right) q^{m} .
$$

When $g$ is holomorphic, this is the usual definition. We will repeatedly use the basic fact that $\mathscr{Y}_{D} g \in M_{2 s}$ if $g \in M_{s+1 / 2}$; see [Kohnen and Zagier 1981]. Recall that a $\mathrm{CM}$ point is a point in $\mathcal{H}$ of the form $\left(-b+\sqrt{b^{2}-4 a c}\right) / 2 a$ for integral $a, b, c$. The proof of the following result will be completed in Section 6. In the case $D=1$, it is due to Borcherds [1998] and follows from a special case of [Theorem 14.3] there; see [Example 14.4].

Theorem 2. For $g \in M_{s+1 / 2}^{!}$with $s \geq 2$ and $D$ a fundamental discriminant with $(-1)^{s} D>0$, the lift $\mathscr{S}_{D} g$ is a meromorphic modular form of weight $2 s$ for $\Gamma$ whose possible poles are of order at most $s$ and occur at CM points.

\section{Weakly holomorphic forms}

In this section we will define a canonical basis for the space $M_{k}^{!}$for any $k=s+1 / 2$ with $s \in \mathbb{Z}$ in which all basis elements have integral Fourier coefficients. Then we will construct forms in $M_{k}^{!}$when $s \geq 2$ using Poincaré series.

We begin by recalling the canonical basis for $M_{2 s}^{!}$defined in [Duke and Jenkins 2008] for any $s \in \mathbb{Z}$. Write $2 s=12 \ell+k^{\prime}$ with uniquely determined $\ell \in \mathbb{Z}$ and $k^{\prime} \in\{0,4,6,8,10,14\}$, so that if $\ell \geq 0$, then $\ell$ is the dimension of the space $S_{2 s}$ of cusp forms of weight $2 s$. For every integer $m \geq-\ell$, there exists a unique $f_{2 s, m} \in M_{2 s}^{!}$with a $q$-expansion of the form $f_{2 s, m}(\tau)=q^{-m}+\sum_{n>\ell} a_{2 s}(m, n) q^{n}$, and together they form a basis for $M_{2 s}^{!}$. The basis element $f_{2 s, m}$ can be given explicitly in the form $f_{2 s, m}=f_{2 s} P(j)$, where $f_{2 s}=f_{2 s,-\ell}=\Delta^{\ell} E_{k^{\prime}}$ and $P$ is a polynomial of degree $m+\ell$. As shown in [Duke and Jenkins 2008], the basis 
elements have the generating function

$$
\sum_{m \geq-\ell} f_{2 s, m}(z) q^{m}=\frac{f_{2 s}(z) f_{2-2 s}(\tau)}{j(\tau)-j(z)}=-\sum_{m \geq \ell+1} f_{2-2 s, m}(\tau) r^{m},
$$

where $r=e(z)$. It follows from this that the coefficients $a_{2 s}(m, n)$ are integral and satisfy the duality relation

$$
a_{2 s}(m, n)=-a_{2-2 s}(n, m) .
$$

In order to formulate a similar result for $M_{k}^{!}$when $k=s+1 / 2$ with $s \in \mathbb{Z}$, let $\ell$ be defined by $2 s=12 \ell+k^{\prime}$ as above. By the Shimura correspondence given in [Kohnen 1980], one finds that the maximal order of a nonzero $f \in M_{k}^{!}$at $i \infty$ is

$$
A= \begin{cases}2 \ell-(-1)^{s} & \text { if } \ell \text { is odd }, \\ 2 \ell & \text { otherwise. }\end{cases}
$$

If $B<A$ is the next admissible exponent we can construct functions in $M_{k}^{!}$of the form

$$
f_{k}(\tau)=q^{A}+O\left(q^{B+4}\right) \quad \text { and } \quad f_{k}^{*}(\tau)=q^{B}+O\left(q^{B+4}\right) .
$$

If we write $s=12 a+b$, where $b \in\{6,8,9,10,11,12,13,14,15,16,17,19\}$, then $f_{k}$ and $f_{k}^{*}$ can be given explicitly in the form

$$
f_{k}(\tau)=\Delta(4 \tau)^{a} f_{b+1 / 2}(\tau) \text { and } f_{k}^{*}(\tau)=\Delta(4 \tau)^{a} f_{b+1 / 2}^{*}(\tau),
$$

where the forms $f_{b+1 / 2}, f_{b+1 / 2}^{*} \in M_{b+1 / 2}$ are given in the appendix and have integral Fourier coefficients. Using them, it is easy to construct a unique basis for $M_{k}^{!}$ consisting of functions of the form

$$
f_{k, m}(\tau)=q^{-m}+\sum_{n>A} a_{k}(m, n) q^{n},
$$

where $m \geq-A$ has $(-1)^{s-1} m \equiv 0,1(\bmod 4)$. Here $f_{k,-A}=f_{k}$ and $f_{k,-B}=f_{k}^{*}$. This can be done recursively: $f_{k, m}(\tau)$ is obtained by multiplying $f_{k, m-4}(\tau)$ by $j(4 \tau)$ and then subtracting a suitable linear combination of the forms $f_{k, m^{\prime}}(\tau)$ with $m^{\prime}<m$. We also have the following generating function, whose proof is similar to Zagier's proof [2002] of the $k=1 / 2$ case:

$$
\sum_{m} f_{k, m}(z) q^{m}=\frac{f_{k}(z) f_{2-k}^{*}(\tau)+f_{k}^{*}(z) f_{2-k}(\tau)}{j(4 \tau)-j(4 z)}=-\sum_{m} f_{2-k, m}(\tau) r^{m} .
$$

This and the fact that $f_{k}$ and $f_{k}^{*}$ have integral Fourier coefficients gives the following result.

Proposition 1. The Fourier coefficients $a_{k}(m, n)$ defined in (8) are integral and satisfy the duality relation

$$
a_{k}(m, n)=-a_{2-k}(n, m) \quad \text { for all } m, n \in \mathbb{Z} .
$$


Another way to construct weakly holomorphic forms is by Poincaré series. Here we will only need them for $k=s+1 / 2$, where $s \geq 2$. Set $j(\gamma, \tau)=\theta(\gamma \tau) / \theta(\tau)$ for $\gamma \in \Gamma_{0}(4)$. For $m \in \mathbb{Z}$, define the Poincaré series

$$
P_{k, m}(\tau)=\sum_{\gamma \in \Gamma_{\infty} \backslash \Gamma_{0}(4)} e(m \gamma \tau) j(\gamma, \tau)^{-2 k}
$$

where $\Gamma_{\infty}$ is the subgroup of translations in $\Gamma_{0}(4)$. For $k \geq 5 / 2$, this series is absolutely convergent and represents a weakly holomorphic form of weight $k$ for $\Gamma_{0}(4)$, but it is not in $M_{k}^{!}$since its Fourier coefficients are not supported on $n$ with $(-1)^{s} n \equiv 0,1(\bmod 4)$. When $m=0$, the Poincaré series is an Eisenstein series that Cohen [1975] projected to a form in $M_{k}$ and whose Fourier coefficients are expressed in terms of the values of Dirichlet $L$-functions at $1-s$. When $m>0$, Kohnen [1985] showed how to obtain in this way cusp forms in $S_{k}$. Bruinier et al. [2006] observed that a similar procedure works for $m<0$. Petersson [1950] had explicitly computed the Fourier expansions of $P_{k, m}$ in terms of Bessel functions and Kloosterman sums, and the projections $g_{k, m}$ of $P_{k, m}$ to $M_{k}^{!}$have Fourier expansions that are simple modifications of these. To give them, for $m, n \in \mathbb{Z}$ and $c \in \mathbb{Z}^{+}$with $c \equiv 0(\bmod 4)$, let

$$
K_{k}(m, n ; c)=\sum_{a(\bmod c)}\left(\frac{c}{a}\right) \varepsilon_{a}^{2 k} e\left(\frac{m a+n \bar{a}}{c}\right)
$$

be the Kloosterman sum, where $\left(\frac{c}{a}\right)$ is the extended Legendre symbol and

$$
\varepsilon_{a}=\left\{\begin{array}{lll}
1 & \text { if } a \equiv 1 \quad(\bmod 4), \\
i & \text { if } a \equiv 3 \quad(\bmod 4)
\end{array}\right.
$$

Also, let $\delta_{\text {odd }}(n)=1$ if $n$ is odd and $\delta_{\text {odd }}(n)=0$ otherwise.

Proposition 2. Suppose $k=s+1 / 2$, where $s \geq 2$. Then, for any nonzero integer $m$ with $(-1)^{s} m \equiv 0,1(\bmod 4)$, there exists a form $g_{k, m} \in M_{k}^{!}$with Fourier expansion

$$
g_{k, m}(\tau)=q^{m}+\sum_{\substack{n \geq 1 \\(-1)^{s} n \equiv 0,1(\bmod 4)}} b_{k}(m, n) q^{n}
$$

where for $(-1)^{s} \equiv 0,1(\bmod 4)$ the coefficient $b_{k}(m, n)$ is given explicitly by the absolutely convergent sum

$$
\begin{aligned}
b_{k}(m, n)=2 \pi i^{-k}\left|\frac{n}{m}\right|^{k-1 / 2} & \sum_{\substack{c>0 \\
c \equiv 0(\bmod 4)}}\left(1+\delta_{\text {odd }}(c / 4)\right) c^{-1} K_{k}(m, n ; c) \\
& \times \begin{cases}I_{k-1}(4 \pi \sqrt{|m n|} / c) & \text { if } m<0, \\
J_{k-1}(4 \pi \sqrt{|m n|} / c) & \text { if } m>0 .\end{cases}
\end{aligned}
$$


When $m=0$, a similar formula holds, and it can be further evaluated to give Cohen's formulas. A modified version holds when $s=1$; see [Bruinier et al. 2006].

Of course, $g_{k,-m}$ can be expressed in terms of the basis elements $f_{k, m}$. If there are no nonzero cusp forms in $M_{2 s}$, then $g_{k,-m}=f_{k, m}$ for all $m$. In general, however,

$$
g_{k,-m}-f_{k, m} \in S_{k}
$$

is a nonzero cusp form. It seems likely that the Fourier coefficients $b_{k}(m, n)$ of $g_{k, m}$ are irrational, even transcendental, in general.

\section{Weak Maass forms}

Next we will show that for $f \in M_{2-2 s}^{!}$with $s \in \mathbb{Z}^{+}$, the function $\partial^{s-1} f$ is a weak Maass form, and we will compute its Fourier expansion. Recall that $\partial^{s-1}$ was defined in (4). Then we express $\partial^{s-1} f_{2-2 s, m}$ in terms of certain Poincaré series. We need the following result which, in essence, is due to Maass; see also [Lewis and Zagier 2001, page 250].

Proposition 3. Suppose $f(\tau)=\sum_{n} a(n) q^{n} \in M_{2-2 s}^{!}$for integral $s \geq 1$. Then $\partial^{s-1} f$ is a weak Maass form for $\Gamma$ with eigenvalue $s(1-s)$. Explicitly, we have

$$
\begin{aligned}
\partial^{s-1} f(\tau)=2 \pi y^{1 / 2} \sum_{n>0} a(-n) n^{s-1 / 2} I_{s-1 / 2}(2 \pi n y) e(-n x) & \\
+(-1)^{s-1}\left(\pi^{1 / 2-s} \Gamma(s-1 / 2) y^{1-s} a(0)\right. & \\
& \left.\quad+2 y^{1 / 2} \sum_{n \neq 0} a(n)|n|^{s-1 / 2} K_{s-1 / 2}(2 \pi|n| y) e(n x)\right),
\end{aligned}
$$

where $I$ and $K$ are the usual Bessel functions.

Proof. By induction it is readily shown that for $n>0$

$$
\partial^{s-1} e(-n \tau)=n^{s-1} \sum_{m=0}^{s-1} \frac{(s-1+m) !}{m !(s-1-m) !}(-4 \pi n y)^{m} e(-n \tau) .
$$

Standard formulas for Bessel functions with half-integral parameter [Gradshteyn and Ryzhik 1994] yield

$$
\begin{aligned}
\partial^{s-1} e(-n \tau) & =2 n^{s-1 / 2} y^{1 / 2}\left(\pi I_{s-1 / 2}(2 \pi n y)+(-1)^{s-1} K_{s-1 / 2}(2 \pi n y)\right) e(n x), \\
\partial^{s-1} e(n \tau) & =2(-1)^{s-1} n^{s-1 / 2} y^{1 / 2} K_{s-1 / 2}(2 \pi n y) e(n x), \\
\partial^{s-1}(1) & =(-1)^{s-1} \pi^{1 / 2-s} \Gamma(s-1 / 2) y^{1-s} .
\end{aligned}
$$

These formulas easily give the stated formula, thus finishing the proof. 
We next express the weak Maass form $\partial^{s-1} f_{2-2 s, m}$ associated to the basis element $f_{2-2 s, m}$ in terms of certain Poincaré series, when $s \geq 2$ and $2 s=12 \ell+k^{\prime}$ as before. For $m \in \mathbb{Z}$ with $m \neq 0$, consider the Poincaré series (see [Niebur 1973])

$$
F_{m}(\tau, s)=2 \pi|m|^{s-1 / 2} \sum_{\gamma \in \Gamma_{\infty} \backslash \Gamma} e(m \operatorname{Re} \gamma \tau)(\operatorname{Im} \gamma \tau)^{1 / 2} I_{s-1 / 2}(2 \pi|m| \operatorname{Im} \gamma \tau),
$$

which converges absolutely for $\operatorname{Re} s>1$. Here $\Gamma_{\infty}$ is the subgroup of translations in $\Gamma$. Clearly $F_{m}(\gamma \tau, s)=F_{m}(\tau, s)$ for $\gamma \in \Gamma$ and $\Delta F_{m}(\tau, s)=s(1-s) F_{m}(\tau, s)$.

Proposition 4. For integral $s \geq 2$, we have for $m \geq \ell+1$

$$
\partial^{s-1} f_{2-2 s, m}(\tau)=F_{-m}(\tau, s)+\sum_{0<n<\ell+1} a_{2-2 s}(m,-n) F_{-n}(\tau, s) .
$$

Proof. We need the Fourier expansion of $F_{m}$. This can be found, for instance, in [Fay 1977]. Let $\xi(s)=\pi^{-s / 2} \Gamma(s / 2) \zeta(s)$. Then we have

$$
\begin{aligned}
F_{m}(\tau, s)=2 \pi|m|^{s-1 / 2} y^{1 / 2} I_{s-1 / 2}(2 \pi|m| y) e(m x)+\frac{4 \pi \sigma_{2 s-1}(|m|)}{(2 s-1) \xi(2 s)} y^{1-s} \\
+4 \pi|m|^{s-1 / 2} \sum_{n \neq 0} c(m, n ; s) y^{1 / 2} K_{s-1 / 2}(2 \pi|n| y) e(n x),
\end{aligned}
$$

where

$$
c(m, n ; s)=\sum_{c>0} c^{-1} K_{0}(m, n ; c) \cdot \begin{cases}I_{2 s-1}\left(4 \pi \sqrt{|m n|} c^{-1}\right) & \text { if } m n<0 \\ J_{2 s-1}\left(4 \pi \sqrt{|m n|} c^{-1}\right) & \text { if } m n>0\end{cases}
$$

and

$$
K_{0}(m, n ; c)=\sum_{a(\bmod c)^{*}} e\left(\frac{m a+n \bar{a}}{c}\right)
$$

is the usual Kloosterman sum, the * restricting the sum to $(a, c)=1$. Consider the Maass form

$$
\phi(\tau)=\partial^{s-1} f_{2-2 s, m}(\tau)-\left(F_{-m}(\tau, s)+\sum_{0<n<\ell+1} a_{2-2 s}(m,-n) F_{-n}(\tau, s)\right) .
$$

By Proposition 3 and (14), we have

$$
\phi(\tau)=c(0) y^{1-s}+\sum_{n \neq 0} c(n) y^{1 / 2} K_{s-1 / 2}(2 \pi|n| y) e(n x),
$$

where each $c(n)$ can be computed explicitly in terms of the $c_{s}(m, n)$ and the $a_{2-2 s}(m, n)$. Since $\phi \in L^{2}(\Gamma \backslash \mathcal{H})$ with eigenvalue $s(1-s)$, it must be equal to 0 .

In the case $s=1$, the Poincaré series $F_{m}(\tau, 1)$ is defined through analytic continuation (see for example [Niebur 1973]), and Proposition 4 continues to hold in the modified form

$$
f_{0, m}(\tau)=j_{m}(\tau)=F_{-m}(\tau, 1)-24 \sigma(m) \quad \text { for } m \geq 1 .
$$




\section{Preliminary formulas for the trace}

For the proof of Theorem 1, we will need to compute the trace of $\partial^{s-1} f_{2-2 s, m}$ in terms of the coefficients of the basis elements $f_{s+1 / 2, m}$. In view of Proposition 4, we are reduced to computing $\operatorname{Tr}_{d, D}\left(F_{m}(\cdot, s)\right)$, where $F_{m}(\tau, s)$ is the Poincaré series defined in (12). When $D=s=1$, it was shown in [Duke 2006] that this trace may be expressed in a simple way in terms of a certain exponential sum. In general we need the exponential sum introduced in [Kohnen 1985]:

$$
S_{m}(d, D ; c)=\sum_{\substack{b(\bmod c) \\ b^{2} \equiv D d(\bmod c)}} \chi\left(\frac{c}{4}, b, \frac{b^{2}-D d}{c}\right) e\left(\frac{2 m b}{c}\right),
$$

where $\chi$ is defined in $(2)$ and $c \equiv 0(\bmod 4)$. Clearly $S_{-m}(d, D ; c)=\overline{S_{m}}(d, D ; c)=$ $S_{m}(d, D ; c)$. We have the following identity.

Proposition 5. Let $s \geq 2$ and $m \neq 0$. Suppose $D$ is fundamental and that $d D<0$. Then

$$
\begin{aligned}
\operatorname{Tr}_{d, D}\left(F_{m}(\cdot, s)\right)=\sqrt{2} \pi|m|^{s-1 / 2}|d|^{1 / 4}|D|^{1 / 4} & \\
& \times \sum_{c \equiv 0(\bmod 4)} c^{-1 / 2} S_{m}(d, D ; c) I_{s-1 / 2}\left(\frac{4 \pi \sqrt{m^{2}|d D|}}{c}\right) .
\end{aligned}
$$

Proof. We have the absolutely convergent expression that

$$
\begin{array}{r}
\operatorname{Tr}_{d, D}\left(F_{m}(\cdot, s)\right)=2 \pi|m|^{s-1 / 2} \sum_{Q} \frac{\chi(Q)}{\omega_{Q}} \sum_{\gamma \in \Gamma_{\infty} \backslash \Gamma} e\left(\begin{array}{r}
\left.\operatorname{Re}_{\gamma} \tau_{Q}\right)\left(\operatorname{Im} \gamma \tau_{Q}\right)^{1 / 2} \\
\times I_{s-1 / 2}\left(2 \pi|m| \operatorname{Im} \gamma \tau_{Q}\right)
\end{array}\right. \\
=\sqrt{2} \pi|m|^{s-\frac{1}{2}}|d|^{1 / 4}|D|^{1 / 4} \sum_{a=1}^{\infty} a^{-1 / 2} I_{s-1 / 2}\left(\frac{\pi \sqrt{m^{2}|d D|}}{a}\right) \\
\times\left[\sum_{Q} \frac{\chi(Q)}{\omega_{Q}} \sum_{\gamma} e\left(m \operatorname{Re}\left(\gamma \tau_{Q}\right)\right)\right],
\end{array}
$$

where the sum over $\gamma$ is over all $\gamma \in \Gamma_{\infty} \backslash \Gamma$ with $\operatorname{Im} \gamma \tau_{Q}=\sqrt{|D d|} /(2 a)$. Consider the sum in brackets in the expression above. For fixed $a>0$, the values of $2 a \operatorname{Re}\left(\gamma \tau_{Q}\right)$ run over the $(\bmod 2 a)$-incongruent solutions to the quadratic congruence $b^{2} \equiv d D(\bmod 4 a)$ with multiplicity $w_{Q}$ as $\gamma$ and $Q$ run over their respective representatives. Thus the term in brackets is equal to $\frac{1}{2} S_{m}(d, D ; 4 a)$. Replacing $4 a$ with $c$ finishes the proof.

We need to express the traces in terms of the Fourier coefficients of modular forms. This is done by applying an identity, originally due to Salié in a special case, to transform the sum of exponential sums in Proposition 5 into a sum of Kloosterman sums. This sum may then be interpreted in terms of the Fourier 
coefficients of half-integral weight Poincaré series. This technique goes back to Zagier [1975], who applied it in the context of base-change. Kohnen [1985] applied it to the Shimura lift of cusp forms. More recently, this method has proved to be fruitful in the context of weakly holomorphic forms. Duke [2006] applied it to give a new proof of Zagier's original identity for traces of singular moduli. The technique has since been extended in various ways in [Jenkins 2006] and [Bringmann and Ono 2007]; in particular, the latter gave the following formula for the trace of $F_{m}(\tau, s)$ in terms of the coefficients $b_{k}(m, n)$ of half-integral weight Poincaré series when $m=-1$ and $(-1)^{s} D<0$.

Proposition 6. Suppose $m \neq 0, s \geq 2$ and $d D<0$ with $D$ fundamental. Then

$$
\begin{aligned}
\operatorname{Tr}_{d, D}\left(F_{m}(\cdot, s)\right)=\varepsilon|d|^{s / 2}|D|^{(1-s / 2)} \sum_{n \mid m} \chi_{D}(n) n^{s-1} b_{s+1 / 2}(-|d|, & \left.\frac{m^{2}|D|}{n^{2}}\right) \\
& \text { if }(-1)^{s} D>0 ;
\end{aligned}
$$

$\operatorname{Tr}_{d, D}\left(F_{m}(\cdot, s)\right)=\varepsilon|d|^{(1-s) / 2}|D|^{s / 2}|m|^{2 s-1} \sum_{n \mid m} \chi_{D}(n) n^{-s} b_{s+1 / 2}\left(\frac{-m^{2}|D|}{n^{2}},|d|\right)$,

$$
\text { if }(-1)^{s} D<0 .
$$

Here the sums $n \mid m$ are over the positive divisors of $m, \varepsilon=(-1)^{\lfloor(s+1) / 2\rfloor}$, and $b_{s+1 / 2}$ was defined in Proposition 2.

Proof. Recall the Kloosterman sum associated to modular forms of half-integral weight defined in (10). It is clear that replacing $k$ with $k+2$ does not change this sum; each $K_{s+1 / 2}(m, n, c)$ is equal to $K_{1 / 2}(m, n ; c)$ or $K_{3 / 2}(m, n ; c)$, depending on whether $s$ is even or odd, respectively. In fact, we have the relations

$$
K_{1 / 2}(m, n ; c)=i \cdot K_{3 / 2}(-m,-n ; c)=K_{1 / 2}(n, m ; c) .
$$

We have the following identity for the Kloosterman sums, which can be proved by a slight modification of the proof of Kohnen [1985, Proposition 5, page 258]; see also [Duke 2006; Jenkins 2006; Tóth 2005].

Lemma 1. For integers $m \neq 0$ and $c>0$ with $4 \mid c$, an integer $d$ with $d \equiv 0,1$ $(\bmod 4)$ and $D$ a fundamental discriminant, we have the identity

$$
S_{m}(d, D ; c)=(1-i) \sum_{n \mid(m, c / 4)}\left(1+\delta_{\text {odd }}(c /(4 n))\right) \chi_{D}(n) \sqrt{\frac{n}{c}} K_{1 / 2}\left(d, m^{2} D / n^{2} ; c / n\right) .
$$

By Proposition 5 and Lemma 1, we quickly derive that

$$
\begin{aligned}
& \operatorname{Tr}_{d, D}\left(F_{m}(\cdot, s)\right)=\sqrt{2} \pi(1-i)|m|^{s-1 / 2}|d|^{1 / 4}|D|^{1 / 4} \sum_{n \mid m} \chi_{D}(n) n^{-1 / 2} \\
& \quad \times \sum_{c \equiv 0(\bmod 4)} c^{-1}\left(1+\delta_{\text {odd }}(c / 4)\right) K_{1 / 2}\left(d, m^{2} D / n^{2} ; c\right) I_{s-1 / 2}\left(\frac{4 \pi}{c} \sqrt{m^{2}|D d| / n^{2}}\right) .
\end{aligned}
$$


Comparison with Proposition 2 and the use of (15) finishes the proof.

\section{The Zagier lift}

In this section we give the proof of Theorem 1. The following proposition gives an explicit formula for the Zagier lift of $f \in M_{2-2 s}^{!}$when $(-1)^{s} D>0$. In its proof we make repeated use of the classical Shimura lift, integral and half-integral weight duality from (7) and (9), and the fact that the constant term of a form in $M_{2}^{!}$must vanish. Write $2 s=12 \ell+k^{\prime}$ with $k^{\prime} \in\{0,4,6,8,10,14\}$ as above.

Proposition 7. Suppose $s \geq 2$ is an integer and $f(\tau)=\sum_{n} a(n) q^{n} \in M_{2-2 s}^{!}$. Suppose $D$ is a fundamental discriminant with $(-1)^{s} D>0$. Then the D-th Zagier lift of $f$ is given by

$$
\mathfrak{Z}_{D} f=\sum_{m>0} a(-m) \sum_{n \mid m} \chi_{D}(n) n^{s-1} f_{3 / 2-s, m^{2}|D| / n^{2}} .
$$

Proof. Recall that when $(-1)^{s} D>0$, the Zagier lift was defined by

$$
\begin{aligned}
\mathfrak{Z}_{D} f(\tau)=\sum_{m>0} a(-m) \sum_{n \mid m} \chi_{D}(n) n^{s-1} q^{-m^{2}|D| / n^{2}} & \\
& +\frac{1}{2} L\left(1-s, \chi_{D}\right) a(0)+\sum_{d: d D<0} \operatorname{Tr}_{d, D}^{*}(f) q^{|d|},
\end{aligned}
$$

where

$$
\operatorname{Tr}_{d, D}^{*}(f)=(-1)^{\lfloor(s-1) / 2\rfloor}|d|^{-s / 2}|D|^{(s-1) / 2} \operatorname{Tr}_{d, D}\left(\partial^{s-1} f\right) .
$$

We prove Proposition 7 by comparing the Fourier coefficients of $\mathfrak{Z}_{D} f$ with those of the function on the right side of (16), which we will denote simply by $F$. We do this separately for the positive coefficients, the principal parts, and the constant terms.

Consider first the positive coefficients. By Propositions 4 and 6 , we have for $m>\ell$ that

$$
\begin{aligned}
-\operatorname{Tr}_{d, D}^{*}\left(f_{2-2 s, m}\right) & =\sum_{n \mid m} \chi_{D}(n) n^{s-1} b_{s+1 / 2}\left(-|d| ; m^{2}|D| / n^{2}\right) \\
+ & \sum_{j=1}^{\ell} a_{2-2 s}(m,-j) \sum_{h \mid j} \chi_{D}(h) h^{s-1} b_{s+1 / 2}\left(-|d| ; j^{2}|D| / h^{2}\right) .
\end{aligned}
$$

From (11), we have the cusp form

$$
C(\tau)=g_{s+1 / 2,-|d|}(\tau)-f_{s+1 / 2,|d|}(\tau)=\sum_{n \geq 1} c(n) q^{n} .
$$

Thus

$$
b_{s+1 / 2}\left(-|d|, j^{2}|D| / h^{2}\right)=a_{s+1 / 2}\left(|d|, j^{2}|D| / h^{2}\right)+c\left(j^{2}|D| / h^{2}\right) .
$$


However, $\mathscr{Y}_{D} C$, the $D$-th Shimura lift of $C$, is a cusp form of weight $2 s$ with $j$-th coefficient $\sum_{h \mid j} \chi_{D}(h) h^{s-1} c\left(j^{2}|D| / h^{2}\right)$. The contribution to $-\operatorname{Tr}_{d, D}^{*}\left(f_{2-2 s, m}\right)$ in (17) from coefficients of $C$, which is

$$
\sum_{n \mid m} \chi_{D}(n) n^{s-1} c\left(m^{2}|D| / n^{2}\right)+\sum_{j=1}^{\ell} a_{2-2 s}(m,-j) \sum_{h \mid j} \chi_{D}(h) h^{s-1} c\left(j^{2}|D| / h^{2}\right),
$$

can be interpreted as the constant term of $\left(\varphi_{D} C\right) f_{2-2 s, m} \in M_{2}^{!}$, which must be zero. Thus we have

$$
\begin{aligned}
-\operatorname{Tr}_{d, D}^{*}\left(f_{2-2 s, m}\right) & =\sum_{n \mid m} \chi_{D}(n) n^{s-1} a_{s+1 / 2}\left(|d|, m^{2}|D| / n^{2}\right) \\
& +\sum_{j=1}^{\ell} a_{2-2 s}(m,-j) \sum_{h \mid j} \chi_{D}(h) h^{s-1} a_{s+1 / 2}\left(|d|, j^{2}|D| / h^{2}\right) .
\end{aligned}
$$

By duality, $\operatorname{Tr}_{d, D}^{*}\left(f_{2-2 s, m}\right)$ is the coefficient of $q^{|d|}$ in the Fourier expansion of

$$
\sum_{n \mid m} \chi_{D}(n) n^{s-1} f_{3 / 2-s, m^{2}|D| / n^{2}}-\sum_{j=1}^{\ell} a_{2 s}(-j, m) \sum_{h \mid j} \chi_{D}(h) h^{s-1} f_{3 / 2-s, j^{2}|D| / h^{2}} .
$$

For an arbitrary form $f=\sum a(m) q^{m} \in M_{2-2 s}^{!}$, we have $f=\sum_{m>\ell} a(-m) f_{2-2 s, m}$, and so

$$
\operatorname{Tr}_{d, D}^{*}(f)=\sum_{m>\ell} a(-m) \operatorname{Tr}_{d, D}^{*}\left(f_{2-2 s, m}\right)
$$

is the coefficient of $q^{|d|}$ in

$$
\begin{aligned}
\sum_{m>\ell} a(-m) & \left(\sum_{n \mid m} \chi_{D}(n) n^{s-1} f_{3 / 2-s, m^{2}|D| / n^{2}}\right. \\
& \left.-\sum_{j=1}^{\ell} a_{2 s}(-j, m) \sum_{h \mid j} \chi_{D}(h) h^{s-1} f_{3 / 2-s, j^{2}|D| / h^{2}}\right) .
\end{aligned}
$$

For $1 \leq j \leq \ell$ we have, once again using that the constant of a form in $M_{2}^{!}$vanishes, that $a(-j)=-\sum_{m>\ell} a(-m) a_{2 s}(-j, m)$. Thus the form in the previous equation simplifies to $F$.

Next consider the principal parts. The properties of the basis elements given in Section 2 show that $f_{3 / 2-s, m^{2}|D| / n^{2}}=0$ if $m^{2}|D| / n^{2}<C$ for some $C$ that depends only on the weight $3 / 2-s$. We use this and the Fourier expansion

$$
f_{3 / 2-s, m^{2}|D| / n^{2}}(\tau)=q^{-m^{2}|D| / n^{2}}+\sum_{h} a_{3 / 2-s}\left(m^{2}|D| / n^{2}, h\right) q^{h}
$$


to write the negative powers of $q$ appearing in the Fourier expansion of $F$ as

$$
\begin{aligned}
& \sum_{m>0} a(-m) \sum_{n \mid m} \chi_{D}(n) n^{s-1} q^{-m^{2}|D| / n^{2}} \\
- & \sum_{m>0} a(-m) \sum_{\substack{n\left|m \\
m^{2}\right| D \mid / n^{2}<C}} \chi_{D}(h) n^{s-1} q^{-m^{2}|D| / n^{2}} \\
+ & \sum_{m, h>0} a(-m) \sum_{n \mid m} \chi_{D}(n) n^{s-1} a_{3 / 2-s}\left(m^{2}|D| / n^{2},-h\right) q^{-h} .
\end{aligned}
$$

The first sum is the principal part of $\mathfrak{Z}_{D} f$, so we must prove that the remaining part, call it $S$, vanishes. By duality,

$$
\begin{aligned}
& S=-\sum_{m>0} a(-m)\left(\sum_{\substack{n\left|m \\
m^{2}\right| D \mid / n^{2}<C}} \chi_{D}(n) n^{s-1} q^{-m^{2}|D| / n^{2}}\right. \\
&\left.+\sum_{h>0} \sum_{n \mid m} \chi_{D}(n) n^{s-1} a_{s+1 / 2}\left(-h, m^{2}|D| / n^{2}\right) q^{-h}\right) .
\end{aligned}
$$

Now for any $h>0$, the coefficient of $q^{m}$ in the Shimura lift $\mathscr{Y}_{D} f_{s+1 / 2,-h}$ of the cusp form $f_{s+1 / 2,-h}$ is given by

$$
\sum_{n \mid m} \chi_{D}(n) n^{s-1} \cdot\left(a_{s+1 / 2}\left(-h, m^{2}|D| / n^{2}\right)+\left\{\begin{array}{ll}
1 & \text { if } m^{2}|D| / n^{2}=h, \\
0 & \text { otherwise. }
\end{array}\right) .\right.
$$

(The last term here arises from the initial $q^{h}$ in the Fourier expansion of $f_{s+1 / 2,-h}$, since $a_{s+1 / 2}(-h, h)$ is zero by definition.) From this, it is clear that the coefficient of $q^{-h}$ in $S$ for each $h>0$ can be interpreted as the constant term of $\left(\mathscr{Y}_{D} f_{s+1 / 2,-h}\right) f \in M_{2}^{!}$, so $S=0$.

Finally we evaluate the constant term of $F$, again using duality, as

$$
\begin{aligned}
\sum_{m>0} a(-m) \sum_{n \mid m} \chi_{D}(n) n^{s-1} a_{3 / 2-s}\left(m^{2}|D| / n^{2}, 0\right) & \\
& =-\sum_{m>0} a(-m) \sum_{n \mid m} \chi_{D}(n) n^{s-1} a_{s+1 / 2}\left(0, m^{2}|D| / n^{2}\right) .
\end{aligned}
$$

Since $s \geq 2$, we have by [Kohnen and Zagier 1981]

$$
\mathscr{Y}_{D} f_{s+1 / 2,0}(\tau)=\frac{1}{2} L\left(1-s, \chi_{D}\right)+\sum_{m>0}\left(\sum_{n \mid m} \chi_{D}(n) h^{s-1} a_{s+1 / 2}\left(0, n^{2}|D| / h^{2}\right)\right) q^{m},
$$

and the constant term of $\left(\mathscr{Y}_{D} f_{s+1 / 2,0}\right) f \in M_{2}^{!}$is

$$
\frac{1}{2} L\left(1-s, \chi_{D}\right) a(0)+\sum_{m>0} a(-m)\left(\sum_{n \mid m} \chi_{D}(n) n^{s-1} a_{s+1 / 2}\left(0, m^{2}|D| / n^{2}\right)\right)=0 .
$$


We also need the corresponding statement if $(-1)^{s} D<0$.

Proposition 8. Suppose $s \geq 2$ is an integer and $f \in M_{2-2 s}^{!}$has Fourier coefficients $a(n)$. Suppose $D$ is a fundamental discriminant with $(-1)^{s} D<0$. Then the $D$-th Zagier lift of $f$ is given by

$$
\mathfrak{Z}_{D} f=\sum_{m>0} a(-m) m^{2 s-1} \sum_{n \mid m} \chi_{D}(n) n^{-s} f_{s+1 / 2, m^{2}|D| / n^{2}}+g,
$$

where $g \in S_{s+1 / 2}$ is the unique cusp form whose Fourier coefficients $b(n)$ match those of $\mathfrak{Z}_{D} f$ for the first $\ell$ positive values of $n$ with $(-1)^{s} n \equiv 0,1(\bmod 4)$.

Proof. Using Propositions 4 and 6 as before, we find that

$$
\begin{array}{r}
\operatorname{Tr}_{d, D}^{*}\left(f_{2-2 s, m}\right)=\sum_{j=1}^{\ell} a_{2-2 s}(m,-j) j^{2 s-1} \sum_{h \mid j} \chi_{D}(h) h^{-s} b_{s+1 / 2}\left(-j^{2}|D| / h^{2} ;|d|\right) \\
+m^{2 s-1} \sum_{n \mid m} \chi_{D}(n) n^{-s} b_{s+1 / 2}\left(-m^{2}|D| / n^{2} ;|d|\right) .
\end{array}
$$

Thus an arbitrary form $f=\sum a(m) q^{m}=\sum_{m>\ell} a(-m) f_{2-2 s, m}$ has trace $\operatorname{Tr}_{d, D}^{*}(f)$ given by

$$
\begin{aligned}
\sum_{m>\ell} a & (-m)\left(m^{2 s-1} \sum_{n \mid m} \chi_{D}(n) n^{-s} b_{s+1 / 2}\left(-m^{2}|D| / n^{2} ;|d|\right)\right. \\
+ & \left.\sum_{j=1}^{\ell} a_{2-2 s}(m,-j) j^{2 s-1} \sum_{h \mid j} \chi_{D}(h) h^{-s} b_{s+1 / 2}\left(-j^{2}|D| / h^{2} ;|d|\right)\right) \\
& =\sum_{m>0} a(-m) m^{2 s-1} \sum_{n \mid m} \chi_{D}(n) n^{-s} b_{s+1 / 2}\left(-m^{2}|D| / n^{2} ;|d|\right)
\end{aligned}
$$

where we have simplified as before. This is just the coefficient of $q^{|d|}$ in the modular form $F \in M_{s+1 / 2}^{!}$given by

$$
F=\sum_{m>0} a(-m) m^{2 s-1} \sum_{n \mid m} \chi_{D}(n) n^{-s} g_{s+1 / 2,-m^{2}|D| / n^{2}}
$$

Now since $g_{s+1 / 2,-m^{2}|D| / n^{2}}-f_{s+1 / 2, m^{2}|D| / n^{2}} \in S_{s+1 / 2}$ from (11), we find that

$$
F=\sum_{m>0} a(-m) m^{2 s-1} \sum_{n \mid m} \chi_{D}(n) n^{-s} f_{s+1 / 2, m^{2}|D| / n^{2}}+g
$$

for a certain cusp form $g$, and, arguing as in Proposition 7 , the principal part of $F$ matches the principal part of $\mathfrak{Z}_{D} f$. Since the constant term and positive coefficients of $F$ match those of $\mathfrak{Z}_{D} f$, Proposition 8 now follows. 
The first statement of Theorem 1 follows from Propositions 7 and 8 . The statement on integrality follows from Proposition 1 in the case $(-1)^{s} D>0$. Otherwise we can reduce to this case using the following identity, which holds if $(-1)^{s} D<0$ and $D^{\prime}$ is fundamental with $(-1)^{s} D^{\prime}>0$ :

$$
\operatorname{Tr}_{m^{2} D^{\prime}, D}^{*}(f)=-m^{2 s-1} \sum_{a \mid m} \mu(a) \chi_{D^{\prime}}(a) \sum_{b \mid m a^{-1}} \chi_{D}(b)(a b)^{-s} \operatorname{Tr}_{(m / a b)^{2} D, D^{\prime}}^{*}(f) .
$$

This identity is a consequence of the following lemma.

Lemma 2. For $D$ and $D^{\prime}$ fundamental discriminants with $D D^{\prime}<0$ and $m \in \mathbb{Z}^{+}$,

$$
\operatorname{Tr}_{m^{2} D^{\prime}, D}=\sum_{a \mid m} \mu(a) \chi_{D^{\prime}}(a) \sum_{b \mid m a^{-1}} \chi_{D}(b) \operatorname{Tr}_{(m / a b)^{2} D, D^{\prime}}
$$

Lemma 2 is obtained by writing the trace as a sum of sums over primitive quadratic forms, noting that $\chi_{D}=\chi_{D^{\prime}}$ for such primitive forms, and applying Möbius inversion.

We now briefly indicate how one shows that the Zagier lift is compatible with the Hecke operators. If $k \in 2 \mathbb{Z}>0$ and $p$ is a prime, the weight $k$ Hecke operator $\left.\right|_{k} T(p)$ acts on a modular form $f(\tau)=\sum_{n} a(n) q^{n} \in M_{k}^{!}$by

$$
\left.f\right|_{k} T(p)=\sum_{n}\left(a(p n)+p^{k-1} a(n / p)\right) q^{n} .
$$

If $k \in 2 \mathbb{Z} \leq 0$, we multiply this by $p^{1-k}$ so that $\left.\right|_{k} T(p)$ preserves the integrality of Fourier coefficients.

When $0<s \in \mathbb{Z}$, the half-integral weight Hecke operator $\left.\right|_{s+1 / 2} T\left(p^{2}\right)$ acts on a form $g(\tau)=\sum_{n} b(n) q^{n} \in M_{s+1 / 2}^{!}$by

$$
\left.g\right|_{s+1 / 2} T\left(p^{2}\right)=\sum_{n}\left(b\left(p^{2} n\right)+\left((-1)^{s} n / p\right) p^{s-1} b(n)+p^{2 s-1} b\left(n / p^{2}\right)\right) q^{n} .
$$

Again, for $s \leq 0$, we normalize this by multiplying by $p^{1-2 s}$.

It is straightforward to see that $\left.\left(\mathfrak{Z}_{D} f\right)\right|_{3 / 2-\hat{s}} T\left(p^{2}\right)=\mathfrak{Z}_{D}\left(\left.f\right|_{2-2 s} T(p)\right)$ for any prime $p$. In the case that $(-1)^{s} D>0$, we need only use the explicit Fourier expansion of the Zagier lift to compare principal parts. If $(-1)^{s} D<0$, though, we must also show that

$$
\begin{array}{r}
\operatorname{Tr}_{(-1)^{s} n, D}^{*}\left(\left.f\right|_{2-2 s} T(p)\right)=\operatorname{Tr}_{(-1)^{s} n p^{2}, D}^{*}(f)+\left((-1)^{s} n / p\right) p^{s-1} \operatorname{Tr}_{(-1)^{s} n, D}^{*}(f) \\
+p^{2 s-1} \operatorname{Tr}_{(-1)^{s} n / p^{2}, D}^{*}(f)
\end{array}
$$

for the first $\ell$ positive values of $n$ with $(-1)^{s} n \equiv 0,1(\bmod 4)$. To see that this holds, we argue as in the proof of [Zagier 2002, Theorem 5(ii)] to show that 
$\operatorname{Tr}_{(-1)^{s} n, D}\left(\left.\left(\partial^{s-1} f\right)\right|_{0} T(p)\right)$ equals

$$
\operatorname{Tr}_{(-1)^{s} n p^{2}, D}\left(\partial^{s-1} f\right)+\left((-1)^{s} n / p\right) \operatorname{Tr}_{(-1)^{s} n, D}\left(\partial^{s-1} f\right)+p \operatorname{Tr}_{(-1)^{s} n / p^{2}, D}\left(\partial^{s-1} f\right),
$$

and we use the fact that if $k<0$, then $\partial_{k}\left(\left.f\right|_{k} T(p)\right)=\left.p \cdot\left(\partial_{k} f\right)\right|_{k+2} T(p)$ to obtain Equation (21).

\section{The Shimura lift}

In this final section we prove Theorem 2. For this we need two propositions:

Proposition 9. Suppose $s \in \mathbb{Z}^{+}$and $\tau \in \mathcal{H}$. As a function of $z \in \mathcal{H}$,

$$
\partial^{s-1}\left(\frac{f_{2 s}(z) f_{2-2 s}(\tau)}{j(\tau)-j(z)}\right)
$$

is a meromorphic modular form of weight $2 s$ with poles of order at most $s$ that only occur at points equivalent to $\tau$ under $\Gamma$.

Proof. Observe first that if $f$ has weight $k$ and $g$ has weight 0 then Equation (3) gives $\partial_{k}(f g)=g \partial_{k}(f)+f \mathcal{D}(g)$. Apply this repeatedly with $g(\tau)=(j(\tau)-$ $j(z))^{-n}$ for $1 \leq n<s$. We derive that

$$
\partial^{s-1}\left(\frac{f_{2-2 s}(\tau)}{j(\tau)-j(z)}\right)=\sum_{n=1}^{s} \frac{g_{n}(\tau)}{(j(z)-j(\tau))^{n}} \quad \text { for } g_{n} \in M_{0}^{!},
$$

from which the result follows easily.

Theorem 2 is a consequence of Proposition 9 together with the following explicit formula for the $D$-th Shimura lift of $f_{s+1 / 2,|d|}$. Write $2 s=12 \ell+k^{\prime}$ as above.

Proposition 10. Suppose $s \geq 2,(-1)^{s} D>0$ and $d D<0$. Then

$$
\mathscr{Y}_{D} f_{s+1 / 2,|d|}(z)=\operatorname{Tr}_{d, D}^{*}\left(\frac{f_{2 s}(z) f_{2-2 s}(\tau)}{j(\tau)-j(z)}\right)+f(z),
$$

where $f \in M_{2 s}$ is the unique holomorphic form whose Fourier coefficients a $(n)$ match those of $\mathscr{Y}_{D} f_{s+1 / 2,|d|}$ for $n=0, \ldots, \ell$.

Proof. By (5) we have, writing $r=e(z)$,

$$
\begin{aligned}
\mathscr{S}_{D} f_{s+1 / 2,|d|}(z)=\frac{1}{2} L\left(1-s, \chi_{D}\right) & a_{s+1 / 2}(|d|, 0) \\
& +\sum_{m>0}\left(\sum_{n \mid m} \chi_{D}(n) n^{s-1} a_{s+1 / 2}\left(|d|, m^{2}|D| / n^{2}\right)\right) r^{m} .
\end{aligned}
$$


By this and (18) we have

$$
\begin{array}{r}
-\sum_{m>\ell} \operatorname{Tr}_{d, D}^{*}\left(f_{2-2 s, m}\right) r^{m}=\mathscr{Y}_{D} f_{s+1 / 2,|d|}(z)-\frac{1}{2} L\left(1-s, \chi_{D}\right) a_{s+1 / 2}(|d|, 0) \\
-\sum_{0<m \leq \ell}\left(\sum_{n \mid m} \chi_{D}(n) n^{s-1} a_{s+1 / 2}\left(|d|, m^{2}|D| / n^{2}\right)\right) r^{m} \\
+\left[\sum_{j=1}^{\ell} \sum_{m>\ell} a_{2-2 s}(m,-j) r^{m} \sum_{h \mid j} \chi_{D}(h) h^{s-1} a_{s+1 / 2}\left(|d|, j^{2}|D| / h^{2}\right)\right] .
\end{array}
$$

Using integral weight duality (7), the bracketed term equals

$$
-\sum_{j=1}^{\ell}\left(f_{2 s,-j}(z)-r^{j}\right) \sum_{h \mid j} \chi_{D}(h) h^{s-1} a_{s+1 / 2}\left(|d|, j^{2}|D| / h^{2}\right),
$$

so the previous equation, after some cancellation, becomes

$$
-\sum_{m>\ell} \operatorname{Tr}_{d, D}^{*}\left(f_{2-2 s, m}\right) r^{m}=\mathscr{Y}_{D} f_{s+1 / 2,|d|}(z)-f(z) .
$$

Then this and (6) imply the claimed identity, at least when $\operatorname{Im} z>\max _{Q} \operatorname{Im} \tau_{Q}$. The full result now follows by analytic continuation.

\section{Acknowledgments}

After this paper was written, we learned that results similar to some of those presented here were obtained independently in [Miller and Pixton 2007]. We thank the referee for some helpful comments.

\section{Appendix}

The table below gives explicit formulas for the first two basis elements $f_{b+1 / 2}$ and $f_{b+1 / 2}^{*}$ of weight $b+1 / 2$ for various $b$ as polynomials in the weight $1 / 2$ theta function $\theta=\sum_{n \in \mathbb{Z}} q^{n^{2}}$ and the weight 2 Eisenstein series on $\Gamma_{0}(4)$ given by $F(z)=$ $\sum_{n=0}^{\infty} \sigma(2 n+1) q^{2 n+1}$. Both $\theta$ and $F$ have integral Fourier coefficients.

The space of holomorphic modular forms on $\Gamma_{0}(4)$ of weight $s+1 / 2$ is generated by the forms $F^{n} \theta^{2 s+1-4 n}$, where $0 \leq n \leq\lfloor(2 s+1 / 4)\rfloor$; see [Cohen 1975]. Thus, in order to construct these basis elements we examine the Fourier expansion of the form $f=\sum_{n=0}^{\lfloor(2 s+1) / 4\rfloor} A(n) F^{n} \theta^{2 s+1-4 n}$ and choose the coefficients $A(n)$ so that $f$ is in the plus space $M_{s+1 / 2}^{!}$and has the appropriate leading terms in its Fourier expansion. The table shows that all of the $A(n)$ are integral for the first two basis elements of each half-integral weight, so it follows that all of the $f_{b+1 / 2}$ and $f_{b+1 / 2}^{*}$ have integral Fourier coefficients. 


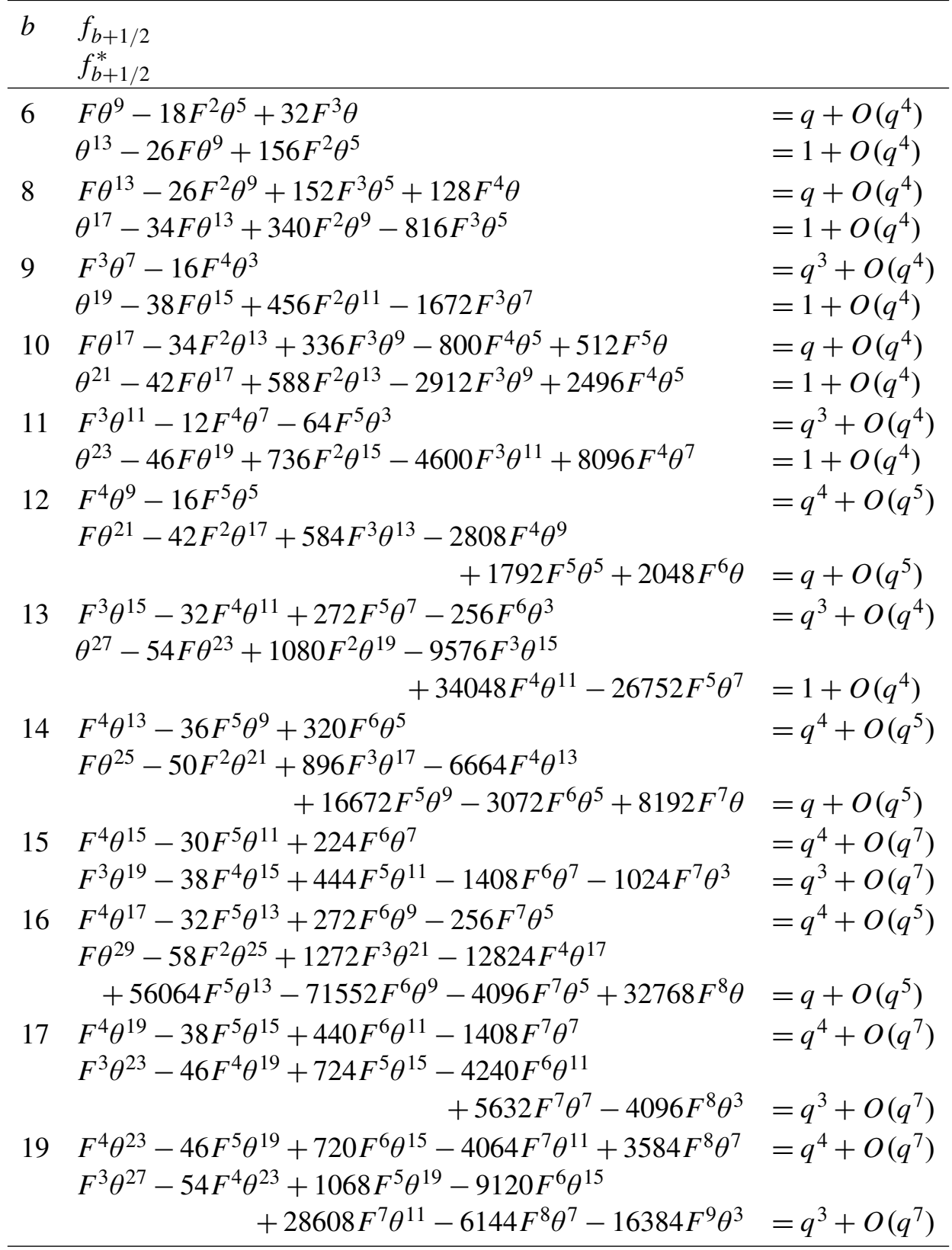

\section{References}

[Borcherds 1995] R. E. Borcherds, "Automorphic forms on $\mathrm{O}_{s+2,2}(\mathbf{R})$ and infinite products", Invent. Math. 120:1 (1995), 161-213. MR 96j:11067 Zbl 0932.11028

[Borcherds 1998] R. E. Borcherds, “Automorphic forms with singularities on Grassmannians", Invent. Math. 132:3 (1998), 491-562. MR 99c:11049 Zbl 0919.11036 
[Bringmann and Ono 2007] K. Bringmann and K. Ono, "Arithmetic properties of coefficients of half-integral weight Maass-Poincaré series”, Math. Ann. 337:3 (2007), 591-612. MR 2007m:11064 Zbl 05145027

[Bruinier and Funke 2006] J. H. Bruinier and J. Funke, "Traces of CM values of modular functions", J. Reine Angew. Math. 594 (2006), 1-33. MR 2007e:11050 Zbl 1104.11021

[Bruinier et al. 2006] J. H. Bruinier, P. Jenkins, and K. Ono, "Hilbert class polynomials and traces of singular moduli”, Math. Ann. 334:2 (2006), 373-393. MR 2007b:11055 Zbl 05013666

[Bruinier et al. 2008] J. H. Bruinier, G. van der Geer, G. Harder, and D. Zagier, The 1-2-3 of modular forms: Lectures at a summer school in Nordfjordeid, Norway, Springer, Berlin, 2008. Zbl 05189476

[Cohen 1975] H. Cohen, "Sums involving the values at negative integers of $L$-functions of quadratic characters", Math. Ann. 217:3 (1975), 271-285. MR 52 \#3080 Zbl 0311.10030

[Duke 2006] W. Duke, "Modular functions and the uniform distribution of CM points", Math. Ann. 334:2 (2006), 241-252. MR 2007e:11051 Zbl 05013661

[Duke and Jenkins 2008] W. Duke and P. Jenkins, "On the zeros and coefficients of certain weakly holomorphic modular forms", Pure Appl. Math. Q. 4:4 (2008), 1327-1340.

[Fay 1977] J. D. Fay, "Fourier coefficients of the resolvent for a Fuchsian group", J. Reine Angew. Math. 293/294 (1977), 143-203. MR 58 \#21944 Zbl 0352.30012

[Funke 2007] J. Funke, "CM points and weight 3/2 modular forms", pp. 107-127 in Analytic number theory: A tribute to Gauss and Dirichlet, edited by W. Duke and Y. Tschinkel, Clay Math. Proc. 7, Amer. Math. Soc., Providence, RI, 2007. MR 2362196 Zbl 05233958

[Gradshteyn and Ryzhik 1994] I. S. Gradshteyn and I. M. Ryzhik, Table of integrals, series, and products, 5th ed., Academic, Boston, 1994. MR 94g:00008 Zbl 0918.65002

[Jenkins 2006] P. Jenkins, "Kloosterman sums and traces of singular moduli”, J. Number Theory 117:2 (2006), 301-314. MR 2006k:11079 Zbl 05023183

[Kohnen 1980] W. Kohnen, "Modular forms of half-integral weight on $\Gamma_{0}(4)$ ", Math. Ann. 248:3 (1980), 249-266. MR 81j:10030 Zbl 0416.10023

[Kohnen 1985] W. Kohnen, "Fourier coefficients of modular forms of half-integral weight", Math. Ann. 271:2 (1985), 237-268. MR 86i:11018 Zbl 0542.10018

[Kohnen and Zagier 1981] W. Kohnen and D. Zagier, "Values of $L$-series of modular forms at the center of the critical strip", Invent. Math. 64:2 (1981), 175-198. MR 83b:10029 Zbl 0468.10015

[Lewis and Zagier 2001] J. Lewis and D. Zagier, "Period functions for Maass wave forms, I", Ann. of Math. (2) 153:1 (2001), 191-258. MR 2003d:11068 Zbl 1061.11021

[Masser 1975] D. Masser, Elliptic functions and transcendence, Lecture Notes in Mathematics 437, Springer, Berlin, 1975. MR 52 \#296 Zbl 0312.10023

[Miller and Pixton 2007] A. Miller and A. Pixton, "Arithmetic traces of non-holomorphic modular invariants", Preprint, 2007, Available at http://www.math.wisc.edu/ ono/reu06zagier.pdf.

[Niebur 1973] D. Niebur, "A class of nonanalytic automorphic functions", Nagoya Math. J. 52 (1973), 133-145. MR 49 \#2557 Zbl 0288.10010

[Petersson 1950] H. Petersson, "Konstruktion der Modulformen und der zu gewissen Grenzkreisgruppen gehörigen automorphen Formen von positiver reeller Dimension und die vollständige Bestimmung ihrer Fourierkoeffizienten", S.-B. Heidelberger Akad. Wiss. Math.-Nat. Kl. 1950 (1950), 417-494. MR 12,806e Zbl 0041.41602

[Ramanujan 1914] S. Ramanujan, "Modular equations and approximations to $\pi$ ", Quart. J. Math. 45 (1914), 350-372. reprinted pp. 23-39 in his Collected papers, AMS/Chelsea, Providence, RI, 2000. MR 2280849 
[Siegel 1969] C. L. Siegel, "Berechnung von Zetafunktionen an ganzzahligen Stellen”, Nachr. Akad. Wiss. Göttingen Math.-Phys. Kl. II 1969 (1969), 87-102. Translated as pp. 249-268 of Advanced analytic number theory, Studies in Mathematics 9, Tata Institute of Fundamental Research, Mumbai, 1980.

[Tóth 2005] Á. Tóth, "On the evaluation of Salié sums”, Proc. Amer. Math. Soc. 133:3 (2005), 643645. MR 2005h:11177 Zbl 02120767

[Zagier 1975] D. Zagier, "Modular forms associated to real quadratic fields", Invent. Math. 30:1 (1975), 1-46. MR 52 \#3062 Zbl 0308.10014

[Zagier 2002] D. Zagier, “Traces of singular moduli”, pp. 211-244 in Motives, polylogarithms and Hodge theory, I (Irvine, CA, 1998), edited by F. Bogomolov and L. Katzarkov, Int. Press Lect. Ser. 3, Int. Press, Somerville, MA, 2002. MR 2004h:11037 Zbl 1048.11035

Communicated by Peter Sarnak

Received 2007-12-03 Revised 2008-05-15 Accepted 2008-06-13

wdduke@ucla.edu

UCLA Mathematics Department, Box 951555, Los Angeles, CA 90095-1555, United States http://www.math.ucla.edu/ wdduke/

jenkins@math.ucla.edu

UCLA Mathematics Department, Box 951555, Los Angeles, CA 90095-1555, United States http://www. math.ucla.edu/ jenkins/ 